\title{
AN ANALYSIS OF THE DARWIN MODEL OF APPROXIMATION TO MAXWELL'S EQUATIONS IN 3-D UNBOUNDED DOMAINS*
}

\author{
CAIXIU LIAO ${ }^{\dagger}$ AND LUNG-AN YING $\ddagger$
}

\begin{abstract}
In this paper, we derive the Darwin model in 3-D unbounded domains by the decomposition of the vector fields; then we show that the Darwin model approximates Maxwell's equations up to the second order for the magnetic flux density, and to the third order for the electric field with respect to $\eta=\frac{\bar{v}}{c}$, where $\bar{v}$ is the characteristic velocity and $c$ is the speed of light.
\end{abstract} model

Key words. Darwin model, Maxwell's equations, 3-D unbounded domains, approximation

AMS subject classifications. 35Q60, 35J25, 35L50

\section{Introduction}

It is known that there are more and more problems involving the solution of Maxwell's equations:

$$
\begin{aligned}
\frac{1}{c^{2}} \frac{\partial \mathbf{E}}{\partial t}-\nabla \times \mathbf{B} & =-\mu J, \\
\frac{\partial \mathbf{B}}{\partial t}+\nabla \times \mathbf{E} & =0, \\
\nabla \cdot \mathbf{E} & =\frac{1}{\varepsilon} \rho, \\
\nabla \cdot \mathbf{B} & =0,
\end{aligned}
$$

where $\mathbf{E}=\mathbf{E}(\mathrm{x}, \mathrm{t}), \mathbf{B}=\mathbf{B}(\mathrm{x}, \mathrm{t})$ denote the electric field and the magnetic flux density respectively, and $\rho=\rho(x, t), \mathbf{J}=\mathbf{J}(x, t)$ are the charge and current densities, respectively, which satisfy the charge conservation equation

$$
\frac{\partial \rho}{\partial t}+\nabla \cdot \mathbf{J}=0
$$

The positive constants $\varepsilon, \mu$ are the electric permittivity and the magnetic permeability of a vacuum, respectively. They are related by

$$
\varepsilon \mu c^{2}=1 \text {. }
$$

In many cases, there are many challenges in dealing with Maxwell's equations directly and the numerical resolution may be very expensive in terms of the computational cost. However, for some problems, e.g., the simulation of charged particle beams when no high frequency phenomenon or no rapid current change occurs, it is possible to use some simplified model which approximates Maxwell's equations and can be solved more economically. The Darwin model is such a simplified model.

*Received: October 30, 2007; accepted (in revised version): January 30, 2008. Communicated by Pierre Degond.

†School of Mathematical Sciences, Xiamen University, Xiamen, 361005, China and Department of Mathematics, South China Normal University, Guangzhou, Guangdong, China (liaocaixiu800919@ 163.com).

${ }^{\ddagger}$ School of Mathematical Sciences, Xiamen University, Xiamen, 361005, China (yingla@xmu. edu.cn). 
In 1992, Degond and Raviart [5] studied the Darwin model in 3-D bounded simply connected domains. In order to derive the Darwin model, they decomposed the electric field $\mathbf{E}$ into the sum of its transverse component $\mathbf{E}_{T}$ and longitudinal component $\mathbf{E}_{L}$, where $\mathbf{E}_{T}$ is divergence free and $\mathbf{E}_{L}$ is curl free. The Darwin model is obtained by neglecting $\frac{\partial \mathbf{E}_{T}}{\partial t}$ in (1.1). They denoted $\mathbf{E}^{D}=\mathbf{E}_{T}^{D}+\mathbf{E}_{L}^{D}$ and $\mathbf{B}^{D}$ as the resulting Darwin approximation to the electric field and magnetic flux density respectively. The Darwin model has the following characteristics (see Degond and Raviart [5]):

(i) $\mathbf{E}_{L}^{D}=\mathbf{E}_{L}=\nabla \psi$, and $\psi$ satisfies

$$
\left\{\begin{array}{l}
\triangle \psi=\frac{\rho}{\varepsilon} \quad \text { in } \quad \Omega, \\
\left.\psi\right|_{\Gamma_{i}}=\alpha_{i}, \quad 0 \leq i \leq m,
\end{array}\right.
$$

where $\alpha_{i}, 0 \leq i \leq m$, are the solutions of the differential system

$$
\left\{\begin{array}{l}
\sum_{j=0}^{m} c_{i j} \frac{d \alpha_{j}}{d t}=\frac{1}{\varepsilon} \int_{\Omega} \mathbf{J} \cdot \nabla \chi_{i} d x, 0 \leq i \leq m \\
\alpha_{i}(0)=\alpha_{i 0}, \quad 0 \leq i \leq m
\end{array}\right.
$$

and $\alpha_{i 0}$ depend on the initial value of $\mathbf{E}_{L}$.

Here $\chi_{i}$ are the solutions of

$$
\left\{\begin{array}{l}
\triangle \chi_{i}=0 \quad \text { in } \Omega \\
\left.\chi_{i}\right|_{\Gamma_{j}}=\delta_{i j}, \quad 0 \leq j \leq m
\end{array}\right.
$$

and $c_{i j}$ stand for $\int_{\Gamma_{j}} \frac{\partial \chi_{i}}{\partial n} d s$.

(ii) $\mathbf{B}^{D}$ satisfies

$$
\begin{aligned}
-\triangle \mathbf{B}^{D} & =\mu \nabla \times \mathbf{J} \quad \text { in } \quad \Omega, \\
\nabla \cdot \mathbf{B}^{D} & =0 \quad \text { in } \quad \Omega, \\
\mathbf{B}^{D} \cdot \mathbf{n} & =\mathbf{B}_{0} \cdot \mathbf{n} \quad \text { on } \quad \partial \Omega, \\
\left(\nabla \times \mathbf{B}^{D}\right) \times \mathbf{n} & =\mu \mathbf{J} \times \mathbf{n} \quad \text { on } \quad \partial \Omega .
\end{aligned}
$$

(iii) $\mathbf{E}_{T}^{D}$ satisfies

$$
\begin{aligned}
& \triangle \mathbf{E}_{T}^{D}=\frac{\partial}{\partial t} \nabla \times \mathbf{B}^{D}, \quad x \in \Omega \\
& \nabla \cdot \mathbf{E}_{T}^{D}=0, \quad x \in \Omega, \\
& \mathbf{E}_{T}^{D} \times\left.\mathbf{n}\right|_{\partial \Omega}=0 \\
& \left\langle\mathbf{E}_{T}^{D} \cdot \mathbf{n}, 1\right\rangle_{\partial \Omega}=0 .
\end{aligned}
$$

Here, $\Gamma_{i}, 0 \leq i \leq m$ are denoted as the connected components of the boundary $\Gamma=\partial \Omega$ with the unit outward normal $\mathbf{n}$, and $\Gamma_{0}$ is the outer boundary. The dual pairing between $H^{-\frac{1}{2}}(\partial \Omega)$ and $H^{\frac{1}{2}}(\partial \Omega)$ is denoted by $\langle\cdot, \cdot\rangle_{\partial \Omega}$.

In 1995, Ciarlet and Zou [6] studied the Darwin model by finite element methods. They derived the $H(\operatorname{curl} ; \Omega)$ and $H(\operatorname{curl}$, div; $\Omega)$ variational formulations for the Darwin model in 3-D bounded simply connected domains, and proved the well-posedness of the variational systems. Nedelec's and standard finite element methods were used to solve these two kinds of variational problems. 
A new approach to deriving the Darwin model in open, bounded, simply connected domains $\Omega \subset R^{3}$, with some generalizations, was introduced by Raviart and Sonnendrücker [8]. Two kinds of boundary conditions were studied, namely, the conditions for the perfect conductor boundary and for the absorbing boundary. The models in [5] are quasi-static, that is, the equations are elliptic and the coefficients might depend on the time $t$. As a result, the initial data in [5] are restricted to a class of functions. On the contrary, the initial data in [8] are general, so the models are governed by evolution equations.

The models in [5] in three dimensional exterior domains were studied by Fang and Ying [3]. Related to the unbounded domains some different spaces were defined and the corresponding variational formulations were studied. Well-posedness was proved. The problems were solved by the infinite element method, and some numerical examples as well as a proof of convergence were provided.

In this paper we study the approximation property of the models in $[5,6,3]$ in three dimensional exterior domains. Being the same as [5] we first derive the Darwin model by using the decomposition result concerning $L^{2}(\Omega)$ in three dimensional unbounded domains. Secondly, we scale the Maxwell's equations, and then we expand the electric field, the magnetic field and the current density in powers of $\eta$. Finally, we show that the approximation of the Darwin model can be analyzed in terms of the dimensionless parameter $\eta$, and the Darwin model approximates Maxwell's equations up to the second order for the magnetic field, and to the third order for the electric field with respect to $\eta$. We consider the perfect conductor boundary only as that in $[5,6]$ and [3]. It is an interesting problem whether the results for quasi-static solutions can be extended to any other boundary conditions, for example the absorbing boundary conditions in [8]. There are some technical difficulties, and this is the subject of our future study.

The contents of the paper are arranged as follows: we first introduce some notations in Section 2; in Section 3 we study the decomposition of the vector fields in 3-D unbounded domains, and we characterize the two parts of the decomposition under suitable boundary conditions. Using the decomposition, we deduce the Darwin model in Section 4. Section 5 is devoted to error estimates: we show that the Darwin model approximates the Maxwell's equations up to the second order for the magnetic flux density, and to the third order for the electric field with respect to $\eta$, which justifies the use of the Darwin model when no rapid change occurs in the physical system.

\section{Notations and preliminaries}

From now on, let $\Omega$ be an open domain in $R^{3}$, and its boundary $\Gamma=\partial \Omega$ be Lipschitz-continuous. When we come to the unbounded problems, we further assume that the boundary $\partial \Omega$ is simply closed. A simply closed curve is a plane curve which is topologically equivalent to (a homeomorphic image of) the unit circle. We denote the complementary domain of $\Omega$ by $\Omega^{c}$. The unit outward normal to $\partial \Omega$ will be denoted by $\mathbf{n}$. Let $x=\left(x_{1}, x_{2}, x_{3}\right)$ be a typical point in $R^{3}$. We define $D(\Omega)$ to be the linear space of infinitely differentiable functions with compact support on $\Omega$, and let $D^{\prime}(\Omega)$ be the dual space of $D(\Omega)$, often called the space of distributions on $\Omega$.

We introduce some Sobolev spaces (see [4]), $L^{2}(\Omega), H^{1}(\Omega), H_{0}^{1}(\Omega), H^{\frac{1}{2}}(\partial \Omega)$, as well as their vector forms $\left(L^{2}(\Omega)\right)^{3},\left(H^{1}(\Omega)\right)^{3},\left(H_{0}^{1}(\Omega)\right)^{3},\left(H^{\frac{1}{2}}(\partial \Omega)\right)^{3}$. If there is no confusion, we use only scalar notation for simplicity. We introduce the inner product $(\cdot, \cdot)$ and the norm $\|\cdot\|_{0}$ in $L^{2}(\Omega) .\|\cdot\|_{1},|\cdot|_{1}$ are the norm and seminorm for $H^{1}(\Omega)$ (also for $H_{0}^{1}(\Omega)$ ). Furthermore, let us introduce some spaces needed for discussing 
our problems. For a bounded domain $\Omega$, the most frequently used Hilbert spaces are

$$
\begin{gathered}
H(\operatorname{div} ; \Omega)=\left\{\mathbf{v} \in\left(L^{2}(\Omega)\right)^{3} ; \nabla \cdot \mathbf{v} \in L^{2}(\Omega)\right\}, \\
H(\operatorname{curl} ; \Omega)=\left\{\mathbf{v} \in\left(L^{2}(\Omega)\right)^{3} ; \nabla \times \mathbf{v} \in\left(L^{2}(\Omega)\right)^{3}\right\},
\end{gathered}
$$

with their subspaces

$$
\begin{gathered}
H_{0}(\operatorname{div} ; \Omega)=\{\mathbf{v} \in H(\operatorname{div} ; \Omega) ; \mathbf{v} \cdot \mathbf{n}=0 \text { on } \quad \Gamma\}, \\
H_{0}(\operatorname{curl} ; \Omega)=\{\mathbf{v} \in H(\operatorname{curl} ; \Omega) ; \mathbf{v} \times \mathbf{n}=0 \text { on } \quad \Gamma\},
\end{gathered}
$$

and their intersection space

$$
H(\operatorname{curl}, \operatorname{div} ; \Omega)=H(\operatorname{div} ; \Omega) \cap H(\operatorname{curl} ; \Omega) .
$$

For the spaces $H(\operatorname{div} ; \Omega), H(\operatorname{curl} ; \Omega)$ and $H(\operatorname{curl}, \operatorname{div} ; \Omega)$, we define the associated norms by

$$
\begin{gathered}
\|\mathbf{v}\|_{0, \operatorname{div}}=\left(\|\mathbf{v}\|_{0}^{2}+\|\nabla \cdot \mathbf{v}\|_{0}^{2}\right)^{\frac{1}{2}}, \\
\|\mathbf{v}\|_{0, \text { curl }}=\left(\|\mathbf{v}\|_{0}^{2}+\|\nabla \times \mathbf{v}\|_{0}^{2}\right)^{\frac{1}{2}}, \\
\|\mathbf{v}\|_{0, \text { curl }, \operatorname{div}}=\left(\|\mathbf{v}\|_{0}^{2}+\|\nabla \cdot \mathbf{v}\|_{0}^{2}+\|\nabla \times \mathbf{v}\|_{0}^{2}\right)^{\frac{1}{2}} .
\end{gathered}
$$

When we come to the exterior problem, the following weighted Sobolev space will be useful (we might assume the origin $o$ is in the interior of $\Omega^{c}$ for simplicity):

$$
H^{1, *}(\Omega)=\left\{\mathbf{u} \in \mathcal{D}^{\prime} ; \nabla \mathbf{u} \in L^{2}(\Omega)^{3}, \frac{\mathbf{u}}{|x|} \in L^{2}(\Omega)^{3}\right\},
$$

which is also a Hilbert space [2], provided with the following norm:

$$
\|\mathbf{u}\|_{1, *}=\left\{\int_{\Omega}|\nabla \mathbf{u}|^{2}+\frac{\mathbf{u}^{2}}{|x|^{2}} d x\right\}^{\frac{1}{2}} .
$$

Let us denote $H_{0}^{1, *}(\Omega)$ as the closure of $C_{0}^{\infty}(\Omega)$ with respect to the norm $|\cdot|_{1}$.

\section{Decomposition of vector fields in 3-D unbounded domains}

We provide the decomposition of the vector fields $\mathbf{f} \in\left(L^{2}(\Omega)\right)^{3}$ in 3-D unbounded domains in this section; we have the following result about the decomposition:

THEOREM 3.1. Any function $\boldsymbol{f} \in\left(L^{2}(\Omega)\right)^{3}$ can be written in the form

$$
\boldsymbol{f}=\nabla \phi+\nabla \times \boldsymbol{u}
$$

where $\phi \in H^{1, *}(\Omega)$ and $\nabla \phi \times\left.\boldsymbol{n}\right|_{\partial \Omega}=0, \boldsymbol{u}$ satisfies $\nabla \cdot \boldsymbol{u}=0$, and

$$
\boldsymbol{u} \in \boldsymbol{B}_{0 c}=\left\{\boldsymbol{u} \in D^{\prime} ;\|\xi \boldsymbol{u}\|_{0, \mathrm{curl}, \mathrm{div}}<\infty,(1-\xi) \boldsymbol{u} \in \boldsymbol{H}_{0}^{1, *}(\Omega),\left.\boldsymbol{u} \cdot \boldsymbol{n}\right|_{\Omega}=0\right\} ;
$$

here $\xi \in C^{\infty}(\Omega)$ is a cutoff function which satisfies: $\xi \equiv 1$ near the boundary $\partial \Omega, \xi \equiv 0$ near infinity, and $0 \leq \xi \leq 1$. 
Proof. First we consider the following problem:

$$
\left\{\begin{array}{l}
\Delta \chi=0 \\
\chi \mid \partial \Omega=1 \\
\lim _{|x| \rightarrow \infty} \chi=0
\end{array}\right.
$$

According to Ying [1] pp. 1-3, we know that the problem (3.1) admits a unique solution in $H^{1, *}(\Omega)$.

Letting $\mathbf{f} \in\left(L^{2}(\Omega)\right)^{3}$, we consider the following problem:

$$
\begin{gathered}
\Delta \phi=\nabla \cdot \mathbf{f}, \\
\left.\phi\right|_{\partial \Omega}=\frac{\int_{\Omega} \mathbf{f} \cdot \nabla \chi d x}{\int_{\partial \Omega} \frac{\partial \chi}{\partial \mathbf{n}} d s}, \\
\lim _{|x| \rightarrow \infty} \phi=0 .
\end{gathered}
$$

According to Ying [1] pp. 13-14, we know that the problem

$$
\int_{\Omega} \nabla \phi \cdot \nabla \mathbf{v} d x=\int_{\Omega} \mathbf{f} \cdot \nabla \mathbf{v} d x \quad, \forall \mathbf{v} \in H_{0}^{1, *}(\Omega)
$$

admits a unique solution in $H_{0}^{1, *}(\Omega)$.

From $(3.2)$, we can get $\nabla \cdot(\nabla \phi-\mathbf{f})=0$. Furthermore, we know that $\nabla \phi-\mathbf{f} \in$ $H(\operatorname{div} ; \Omega)$ and $\left.(\nabla \phi-\mathbf{f}) \cdot \mathbf{n}\right|_{\partial \Omega} \in H^{-\frac{1}{2}}(\partial \Omega)$.

We take a sequence $\left\{\mathbf{f}_{n}\right\}$ such that $\left\{\mathbf{f}_{n}\right\} \rightarrow \mathbf{f}$ in $\left(L^{2}(\Omega)\right)^{3}$, where $\left\{\mathbf{f}_{n}\right\} \in C^{\infty}(\Omega)^{3}$ and $\operatorname{supp}\left\{\mathbf{f}_{n}\right\} \subset \subset \Omega$. From the sequence $\left\{\mathbf{f}_{n}\right\}$ we can get a sequence $\left\{\phi_{n}\right\}$. We let $\Omega_{1}=\Omega \cap B(0, R)$, and $\operatorname{supp}\left\{\mathbf{f}_{n}\right\} \subset B(0, R)$.

$$
\begin{aligned}
& \int_{\Omega_{1}} \Delta \phi_{n} \chi d x=\int_{\partial \Omega}\left(\frac{\partial \phi_{n}}{\partial \mathbf{n}} \chi-\frac{\partial \chi}{\partial \mathbf{n}} \phi_{n}\right) d s-\int_{\partial B(0, R)}\left(\frac{\partial \phi_{n}}{\partial \mathbf{n}} \chi-\frac{\partial \chi}{\partial \mathbf{n}} \phi_{n}\right) d s \\
& \rightarrow \int_{\partial \Omega}\left(\frac{\partial \phi_{n}}{\partial \mathbf{n}} \chi-\frac{\partial \chi}{\partial \mathbf{n}} \phi_{n}\right) d s \quad(R \rightarrow \infty) . \\
& \int_{\Omega_{1}}\left(\nabla \cdot \mathbf{f}_{n}\right) \chi d x=-\int_{\Omega_{1}} \mathbf{f}_{n} \cdot \nabla \chi d x+\int_{\partial \Omega} \mathbf{f}_{n} \chi \cdot \mathbf{n} d s+\int_{\partial B(0, R)} \mathbf{f}_{n} \chi \cdot \mathbf{n} d s \\
& \rightarrow-\int_{\Omega}^{\mathbf{f}_{n} \cdot \nabla \chi d x+\int_{\partial \Omega} \mathbf{f}_{n} \chi \cdot \mathbf{n} d s \quad(R \rightarrow \infty) .}
\end{aligned}
$$

Combining (3.6), (3.7) with the fact that $\int_{\partial \Omega} \frac{\partial \chi}{\partial \mathbf{n}} \phi_{n} d s=\int_{\Omega} \mathbf{f}_{n} \cdot \nabla \chi d x$, and letting $n \rightarrow \infty$, we can get

$$
\int_{\partial \Omega}(\nabla \phi-\mathbf{f}) \cdot \mathbf{n} d s=0 .
$$

(a) Now we consider the function $\mathbf{f}$ satisfying: $\nabla \times \mathbf{f}=0, \mathbf{f} \times\left.\mathbf{n}\right|_{\partial \Omega}=0$.

From $\nabla \times \mathbf{f}=0$, we can get

$$
\nabla \times(\nabla \phi-\mathbf{f})=0
$$


Combining (3.3) with $\mathbf{f} \times\left.\mathbf{n}\right|_{\partial \Omega}=0$, we can obtain:

$$
(\nabla \phi-\mathbf{f}) \times\left.\mathbf{n}\right|_{\partial \Omega}=0 .
$$

Combining with (3.2), (3.8), (3.9) and (3.10), according to Fang and Ying [3], we obtain $\nabla \phi-\mathbf{f}=0$. So $\mathbf{f}=\nabla \phi$.

(b) Now we consider the function $\mathbf{f}$ satisfying: $\nabla \cdot \mathbf{f}=0, \int_{\partial \Omega} \mathbf{f} \cdot \mathbf{n} d s=0$.

We consider the following variational problem (3.11-3.12):

$$
\begin{gathered}
\int_{\Omega}(\nabla \times \mathbf{u})(\nabla \times \mathbf{v}) d x+\int_{\Omega}(\nabla \cdot \mathbf{v}) p d x=\int_{\Omega} \mathbf{f} \cdot(\nabla \times \mathbf{v}) d x, \quad \forall \mathbf{v} \in \mathbf{B}_{0 c} \\
\int_{\Omega}(\nabla \cdot \mathbf{u}) q d x=0, \forall q \in L^{2}(\Omega) .
\end{gathered}
$$

According to Fang and Ying [3], the problem (3.11)-(3.12) admits a unique solution $\mathbf{u} \in \mathbf{B}_{0 c}, \nabla \cdot \mathbf{u}=0$, and $p=0$.

Notice that since $p=0$, it follows from (3.11) that we can get

$$
\nabla \times(\mathbf{f}-\nabla \times \mathbf{u})=0
$$

and

$$
(\mathbf{f}-\nabla \times \mathbf{u}) \times\left.\mathbf{n}\right|_{\partial \Omega}=0 .
$$

Applying the result of (i), we obtain $\mathbf{f}-\nabla \times \mathbf{u}=\nabla \phi$. From $\nabla \cdot \mathbf{f}=0$, combining with (3.2), we get $\Delta \phi=0$.

We take a sequence $\left\{\chi_{n}\right\} \in C^{\infty}(\Omega)$ such that $\left\{\chi_{n}\right\} \rightarrow \chi$ in $H^{1, *}(\Omega)$. According to inregration by parts, we have

$$
\int_{\Omega} \mathbf{f} \cdot \nabla \chi_{n} d x=\int_{\partial \Omega} \mathbf{f} \cdot \chi_{n} \mathbf{n} d s
$$

Combining with $\int_{\partial \Omega} \mathbf{f} \cdot \mathbf{n} d s=0$, and letting $n \rightarrow \infty$, we can get

$$
\int_{\Omega} \mathbf{f} \cdot \nabla \chi d x=0 .
$$

Next we extend $\chi$ such that $\chi \equiv 1$ for $x \in \Omega^{c}$. According to the extention, we know that $\nabla \chi=0$ in $\Omega^{c}$. So we have

$$
\int_{\Omega}(\nabla \times \mathbf{u}) \cdot(\nabla \chi) d x=\int_{R^{3}}(\nabla \times \mathbf{u}) \cdot(\nabla \chi) d x .
$$

Since $\chi_{n} \rightarrow \chi$ in $H^{1, *}(\Omega)$ and $\chi_{n} \in C_{0}^{\infty}\left(R^{3}\right)$, we have

$$
\int_{R^{3}}(\nabla \times \mathbf{u}) \cdot\left(\nabla \chi_{n}\right) d x=-\int_{R^{3}} \nabla \cdot(\nabla \times \mathbf{u}) \chi_{n} d x=0 .
$$

Exactly speaking, the formula (3.18) is obtained using Green's formula in a big enough ball. 
Letting $n \rightarrow \infty$, then we can get

$$
\int_{R^{3}}(\nabla \times \mathbf{u}) \cdot(\nabla \chi) d x=0
$$

Combining (3.19) with (3.16), we can get

$$
\int_{\Omega}(\mathbf{f}-\nabla \times \mathbf{u}) \cdot(\nabla \chi) d x=0 .
$$

Therefore, when the function $\mathbf{f}$ satisfies $\nabla \cdot \mathbf{f}=0, \int_{\partial \Omega} \mathbf{f} \cdot \mathbf{n} d s=0$; combining with (3.3), we have

$$
\left.\phi\right|_{\partial \Omega}=\frac{\int_{\Omega}(\mathbf{f}-\nabla \times \mathbf{u}) \cdot \nabla \chi d x}{\int_{\partial \Omega} \frac{\partial \chi}{\partial \mathbf{n}} d s},
$$

i.e., $\left.\phi\right|_{\partial \Omega}=0$. Combining with $\Delta \phi=0$ and (3.4), we have $\phi=0$. According to $\mathbf{f}-\nabla \times \mathbf{u}=\nabla \phi$, we have $\mathbf{f}=\nabla \times \mathbf{u}$.

(c) For the general function $\mathbf{f} \in\left(L^{2}(\Omega)\right)^{3}$ :

We consider the following problem:

$$
\begin{gathered}
\nabla \cdot(\mathbf{f}-\nabla \phi)=0, \\
\int_{\partial \Omega}(\mathbf{f}-\nabla \phi) \cdot \mathbf{n} d s=0,
\end{gathered}
$$

where $\phi$ is the solution of the problem (3.2)-(3.4).

According to the result of (ii), there exists a unique $\mathbf{u}$ such that $\mathbf{f}-\nabla \phi=\nabla \times \mathbf{u}$, where $\mathbf{u} \in \mathbf{B}_{0 c}$ and $\mathbf{u}$ satisfies $\nabla \cdot \mathbf{u}=0$. So Theorem 3.1 holds.

\section{The Darwin model in 3-D unbounded domains}

Now we consider Maxwell's equations (1.1)-(1.4) in $\Omega \times(0, T)$ with the following boundary conditions:

$$
\begin{gathered}
\mathbf{E} \times\left.\mathbf{n}\right|_{\partial \Omega}=0, \\
\left.\frac{\partial}{\partial t} \mathbf{B} \cdot \mathbf{n}\right|_{\partial \Omega}=0,
\end{gathered}
$$

and the initial conditions:

$$
\begin{aligned}
& \mathbf{E}(\cdot, 0)=\mathbf{E}_{0}, \\
& \mathbf{B}(\cdot, 0)=\mathbf{B}_{0} ;
\end{aligned}
$$

the initial data $\mathbf{E}_{0}, \mathbf{B}_{0}$ satisfy the constraints:

$$
\begin{gathered}
\nabla \cdot \mathbf{E}_{0}=\frac{\rho_{0}}{\varepsilon} \text { in } \Omega, \quad \rho_{0}=\rho(\cdot, 0), \\
\nabla \cdot \mathbf{B}_{0}=0 \text { in } \Omega,
\end{gathered}
$$




$$
\mathbf{E}_{0} \times\left.\mathbf{n}\right|_{\Gamma}=0
$$

The well-posedness of the initial boundary value problem of Maxwell's equations (1.1)-(1.4), (4.1)-(4.4) was shown in [1].

We define $\mathbf{u}=\left(\begin{array}{c}\mathbf{E} \\ \mathbf{H}\end{array}\right) ; \mathbf{H}=\left(H_{1}, H_{2}, H_{3}\right)^{T}$ is the magnetic field and $\mathbf{B}=\mu \mathbf{H}$.

We first define

$$
A=-i\left(\begin{array}{cc}
\varepsilon^{-1} & 0 \\
0 & \mu^{-1}
\end{array}\right)\left(\begin{array}{ll}
0 & -\nabla \times \\
\nabla \times & 0
\end{array}\right)
$$

The space is

$$
D(A)=H_{0}(\operatorname{curl} ; \Omega) \times H(\operatorname{curl} ; \Omega) .
$$

The initial boundary value problem can be written in the form of

$$
\frac{d \mathbf{u}}{d t}+i A \mathbf{u}=\mathbf{f}, \quad \mathbf{u}(0)=\mathbf{u}_{0},
$$

where $\mathbf{f}=\left(\begin{array}{l}-\frac{1}{\varepsilon} J \\ 0\end{array}\right)$

Then we define a weight

$$
w=\left(\begin{array}{cc}
\varepsilon & 0 \\
0 & \mu
\end{array}\right)
$$

and a weighted Hilbert space $\mathbf{L}_{w}^{2}(\Omega)$ with respect to the inner product $(\cdot, \cdot)_{w}=(w \cdot, \cdot)$ and norm $\|\cdot\|_{0, w}$. We choose $H_{w}=\mathbf{L}_{w}^{2}(\Omega)^{3} \times \mathbf{L}_{w}^{2}(\Omega)^{3}$; according to [1] p. 57, we know the operator $A: D(A) \rightarrow H_{w}$ is a self-adjoint operator.

We assume that $\mathbf{f} \in C\left([0, \infty) ; H_{w}\right)$, the formulation of a weak solution to the problem (4.8) is: Find $\mathbf{u} \in C\left([0, \infty) ; H_{w}\right)$ such that

$$
\int_{0}^{\infty}\left\{\left(\mathbf{u},-\frac{d \varphi}{d t}+i A \varphi\right)_{w}-(\mathbf{f}, \varphi)_{w}\right\} d t-\left(\mathbf{u}_{0}, \varphi(\cdot, 0)\right)_{w}=0
$$

for all $\varphi=\left(\begin{array}{l}\varphi_{1} \\ \varphi_{2}\end{array}\right) \in C_{0}([0, \infty) ; D(A)) \cap C^{1}\left([0, \infty) ; H_{w}\right)$.

According to Theorem 38 in [1], the problem (4.9) admits a unique solution $\mathbf{u} \in C^{1}\left([0, \infty) ; H_{w}\right)$.

Theorem 4.1. Assume $A \boldsymbol{u}_{0} \in H_{w}$ and $\int_{0}^{t}\|A \boldsymbol{f}\|_{0, w} d \tau<\infty$; then $A \boldsymbol{u} \in L^{\infty}\left((0, T) ; H_{w}\right)$, $\forall T>0$.

Proof. The proof is similar to the proof of Theorem 4.1 in [7], so we omit the details here.

Theorem 4.2. Assume $\nabla \cdot \boldsymbol{E}_{0} \in L^{2}(\Omega)$, then $\nabla \cdot \boldsymbol{E} \in L^{2}\left((0, \infty) ; L^{2}(\Omega)\right)$.

Proof. We let $\varphi_{2}=0$ in the formula (4.9); then the formula (4.9) becomes the following:

$$
\int_{0}^{\infty} \int_{\Omega}\left\{-\mathbf{E} \frac{\partial \varphi_{1}}{\partial t}-\frac{1}{\varepsilon} \mathbf{H} \cdot \nabla \times \varphi_{1}\right\} d x d t-\int_{\Omega} \mathbf{E}_{0}(x) \cdot \varphi_{1}(x, 0) d x=\int_{0}^{\infty} \int_{\Omega}-\frac{1}{\varepsilon} \mathbf{J} \cdot \varphi_{1} d x d t
$$


Now we take $\varphi_{1}=\nabla \phi$, where $\phi \in C^{1}\left([0, \infty) ; C_{0}^{\infty}(\Omega)\right)$, then the formula (4.10) turns to be the following:

$$
\int_{0}^{\infty} \int_{\Omega}\left\{-\mathbf{E} \frac{\partial \nabla \phi}{\partial t}\right\} d x d t-\int_{\Omega} \mathbf{E}_{0}(x) \cdot \nabla \phi(x, 0) d x=\int_{0}^{\infty} \int_{\Omega}-\frac{1}{\varepsilon} \mathbf{J} \cdot \nabla \phi d x d t .
$$

From $\nabla \cdot \mathbf{E}_{0} \in L^{2}(\Omega)$ we have $\nabla \cdot \mathbf{E} \in L^{2}\left((0, \infty) ; L^{2}(\Omega)\right)$.

Theorem 4.3. Assume $\nabla \cdot \boldsymbol{B}_{0} \in L^{2}(\Omega)$; then $\nabla \cdot \boldsymbol{B} \in L^{2}\left((0, \infty) ; L^{2}(\Omega)\right)$.

The proof is the same as for Theorem 4.2.

We know Maxwell's equations (1.1)-(1.4) with the boundary conditions (4.1)(4.2) and the initial conditions (4.3)-(4.4) are well-posed. Combining with Theorem 4.1, Theorem 4.2 and Theorem 4.3, we know that there exists a strong solution of Maxwell's equations. Now if we neglect $\frac{\partial \mathbf{E}_{T}}{\partial t}$ in (1.1), then the approximations $\mathbf{E}^{D}=$ $\mathbf{E}_{T}^{D}+\mathbf{E}_{L}^{D}, \mathbf{B}^{D}$ of $\mathbf{E}, \mathbf{B}$ are the solutions of the following equations:

$$
\begin{gathered}
\frac{1}{c^{2}} \frac{\partial \mathbf{E}_{L}^{D}}{\partial t}-\nabla \times \mathbf{B}^{D}=-\mu J, \\
\frac{\partial \mathbf{B}^{D}}{\partial t}+\nabla \times \mathbf{E}_{T}^{D}=0, \\
\nabla \cdot \mathbf{E}_{T}^{D}=0, \\
\nabla \cdot \mathbf{E}_{L}^{D}=\frac{\rho}{\varepsilon}, \\
\nabla \cdot \mathbf{B}^{D}=0,
\end{gathered}
$$

and the boundary conditions satisfy the following:

$$
\begin{aligned}
& \mathbf{E}_{T}^{D} \times\left.\mathbf{n}\right|_{\partial \Omega}=0, \\
& \mathbf{E}_{L}^{D} \times\left.\mathbf{n}\right|_{\partial \Omega}=0, \\
& \int_{\partial \Omega} \mathbf{E}_{T}^{D} \cdot \mathbf{n} d s=0, \\
& \mathbf{B}^{D} \cdot \mathbf{n}=\mathbf{B}_{0} \cdot \mathbf{n} .
\end{aligned}
$$

Combining with (4.13), (4.14), (4.17) and (4.19), we know that $\mathbf{E}_{T}^{D}$ satisfies

$$
\left\{\begin{array}{l}
\nabla \times \mathbf{E}_{T}^{D}=-\frac{\partial \mathbf{B}^{D}}{\partial t}, \\
\nabla \cdot \mathbf{E}_{T}^{D}=0, \\
\mathbf{E}_{T}^{D} \times\left.\mathbf{n}\right|_{\partial \Omega}=0 \\
\int_{\partial \Omega} \mathbf{E}_{T}^{D} \cdot \mathbf{n} d s=0 .
\end{array}\right.
$$


From (4.12), combining with the decomposition of the vector fields,

$$
-\triangle \mathbf{B}^{D}=\mu \nabla \times \mathbf{J}
$$

and

$$
\nabla \times \mathbf{B}^{D} \times\left.\mathbf{n}\right|_{\partial \Omega}=\mu \mathbf{J} \times \mathbf{n} .
$$

Combining with (4.22), (4.23), (4.16), (4.20), $\mathbf{B}^{D}$ satisfies

$$
\left\{\begin{array}{l}
-\triangle \mathbf{B}^{D}=\mu_{0} \nabla \times \mathbf{J}, \\
\nabla \cdot \mathbf{B}^{D}=0 \\
\mathbf{B}^{D} \cdot \mathbf{n}=\mathbf{B}_{0} \cdot \mathbf{n}, \\
\nabla \times \mathbf{B}^{D} \times\left.\mathbf{n}\right|_{\partial \Omega}=\mu_{0} \mathbf{J} \times \mathbf{n} .
\end{array}\right.
$$

According to $\nabla \times \mathbf{E}_{L}^{D}=0, \mathbf{E}_{L}^{D} \times\left.\mathbf{n}\right|_{\partial \Omega}=0$, there exists a function $\phi$ such that $\mathbf{E}_{L}^{D}=$ $\nabla \phi$; combining with (4.12), we can see that $\frac{\partial \phi}{\partial t}$ is the solution of

$$
\left\{\begin{array}{l}
\frac{\partial}{\partial t} \triangle \phi=-\frac{1}{\varepsilon} \nabla \cdot \mathbf{J} \\
\left.\phi\right|_{\partial \Omega}=-\frac{\frac{1}{\varepsilon} \int_{\Omega} \mathbf{J} \cdot \nabla \chi d x}{\int_{\partial \Omega} \frac{\partial \chi}{\partial \mathbf{n}} d s} \\
\lim _{|x| \rightarrow \infty} \phi=0 .
\end{array}\right.
$$

Combining with the charge conservation equation (1.5) and the hypothesis that $\triangle \phi(\cdot, 0)=\frac{\rho_{0}}{\varepsilon}$, we can obtain the following:

$$
\left\{\begin{array}{l}
\triangle \phi=\frac{\rho}{\varepsilon}, \\
\left.\phi\right|_{\partial \Omega}=-\frac{\frac{1}{\varepsilon} \int_{\Omega} \mathbf{J} \cdot \nabla \chi d x}{\int_{\partial \Omega} \frac{\partial \chi}{\partial \mathbf{n}} d s}, \\
\lim _{|x| \rightarrow \infty} \phi=0 .
\end{array}\right.
$$

The problem (4.26) is a Poisson equation with Dirichlet boundary condition, so it is easy to know that the problem (4.26) is well-posed. According to Fang and Ying [3], the problem (4.21) and the problem (4.24) are well-posed. So after neglecting $\frac{\partial \mathbf{E}_{T}}{\partial t}$ in Maxwell's equation, the problem (4.12)-(4.20) is still well-posed.

\section{Error estimates}

First we claim that $\mathbf{E}_{L}^{D}=\mathbf{E}_{L}$. In order to explain this result clearly, we introduce some lemmas first:

LEMmA 5.1. Let $\boldsymbol{f} \in\left(L^{2}(\Omega)\right)^{3}$. Then $\nabla \times \boldsymbol{f}=0$ in $\Omega, \boldsymbol{f} \times\left.\boldsymbol{n}\right|_{\partial \Omega}=0$ if and only if there exists a function $\phi \in H^{1, *}(\Omega)$ such that $\boldsymbol{f}=\nabla \phi$; moreover, the function $\phi$ satisfies (3.2-3.4).

Proof. The sufficiency is obvious, and the necessity follows by Theorem 3.1 (i).

Lemma 5.2. Let $\boldsymbol{f} \in\left(L^{2}(\Omega)\right)^{3}$. Then $\nabla \cdot \boldsymbol{f}=0$ in $\Omega, \int_{\partial \Omega} \boldsymbol{f} \cdot \boldsymbol{n} d s=0$ if and only if there exists a function $\boldsymbol{u}$ such that $\boldsymbol{f}=\nabla \times \boldsymbol{u}$, where $\boldsymbol{u} \in \boldsymbol{B}_{0 c}$ and $\nabla \cdot \boldsymbol{u}=0$.

Proof. The sufficiency is obvious, and the necessity follows by Theorem 3.1 (ii).. 
Next we introduce the following subspaces of the space $L^{2}(\Omega)^{3}$.

$$
\begin{gathered}
\mathbf{L}_{1}=\left\{\mathbf{f} \in L^{2}(\Omega)^{3} ; \nabla \cdot \mathbf{f}=0, \quad \int_{\partial \Omega} \mathbf{f} \cdot \mathbf{n} d s=0\right\}, \\
\mathbf{L}_{2}=\left\{\mathbf{f} \in L^{2}(\Omega)^{3} ; \nabla \times \mathbf{f}=0, \quad \mathbf{f} \times\left.\mathbf{n}\right|_{\partial \Omega}=0\right\} .
\end{gathered}
$$

TheOREm 5.3. Assume that the domain $\Omega$ is 3-dimensional and unbounded; then we have the following decomposition of the space $L^{2}(\Omega)^{3}: L^{2}(\Omega)^{3}=\boldsymbol{L}_{1} \oplus \boldsymbol{L}_{2}$.

Proof. We let

$$
\begin{gathered}
\widetilde{\mathbf{L}_{1}}=\left\{\nabla \times \mathbf{u} ; \mathbf{u} \in \mathbf{B}_{0 c}, \nabla \cdot \mathbf{u}=0\right\}, \\
\widetilde{\mathbf{L}_{2}}=\left\{\nabla \phi, \phi \in H^{1, *}(\Omega) ; \phi \text { satisfying }(3.2)--(3.4)\right\} .
\end{gathered}
$$

Use Green's formula:

$$
\int_{\Omega}(\nabla \times \mathbf{u}) \cdot \nabla \phi d x=\int_{\Gamma}(\mathbf{n} \times \mathbf{u}) \cdot \nabla \phi d s=\int_{\Gamma} \mathbf{u} \cdot(\nabla \phi \times \mathbf{n}) d s=0 .
$$

We know that $\widetilde{\mathbf{L}_{1}}, \widetilde{\mathbf{L}_{2}}$ are orthogonal subspaces of $L^{2}(\Omega)^{3}$; according to the decomposition of the vector fields, we know that $L^{2}(\Omega)^{3}=\widetilde{\mathbf{L}_{1}} \oplus \widetilde{\mathbf{L}_{2}}$.

Furthermore, according to Lemma 5.1 and Lemma 5.2, we know $\widetilde{\mathbf{L}_{1}}=\mathbf{L}_{1}, \widetilde{\mathbf{L}_{2}}=\mathbf{L}_{2}$, so $L^{2}(\Omega)^{3}=\mathbf{L}_{1} \oplus \mathbf{L}_{2}$.

Theorem 5.4. Assume $\boldsymbol{E}_{L}^{D}(\cdot, 0)=\boldsymbol{E}_{0 L}$; then we have $\boldsymbol{E}_{L}^{D}=\boldsymbol{E}_{L}$.

Proof. Comparing (1.1) with (4.12), we have

$$
\mu J=\nabla \times \mathbf{B}-\frac{1}{c^{2}} \frac{\partial \mathbf{E}_{T}}{\partial t}-\frac{1}{c^{2}} \frac{\partial \mathbf{E}_{L}}{\partial t}=\nabla \times \mathbf{B}^{D}-\frac{1}{c^{2}} \frac{\partial \mathbf{E}_{L}^{D}}{\partial t} .
$$

We find that $\nabla \times \mathbf{B}-\frac{1}{c^{2}} \frac{\partial \mathbf{E}_{T}}{\partial t}, \nabla \times \mathbf{B}^{D} \in \mathbf{L}_{1}$, and $\frac{1}{c^{2}} \frac{\partial \mathbf{E}_{L}}{\partial t}, \frac{1}{c^{2}} \frac{\partial \mathbf{E}_{L}^{D}}{\partial t} \in \mathbf{L}_{2}$.

Using the uniqueness of the decomposition of vector fields given by Theorem 5.3, we know $\frac{\partial \mathbf{E}_{L}^{D}}{\partial t}=\frac{\partial \mathbf{E}_{L}}{\partial t}$. Combining with $\mathbf{E}_{L}^{D}(\cdot, 0)=\mathbf{E}_{0 L}$, we have $\mathbf{E}_{L}^{D}=\mathbf{E}_{L}$. $\mathbf{B}^{D}-\mathbf{B}$.

We know already that $\mathbf{E}_{L}^{D}=\mathbf{E}_{L}$; it remains to evaluate the errors $\mathbf{E}_{T}^{D}-\mathbf{E}_{T}$ and

We introduce

$$
\begin{aligned}
& \bar{l}=\text { characteristic length, } \\
& \bar{t}=\text { characteristic time, } \\
& \bar{v}=\overline{\bar{l}}=\text { characteristic velocity, } \\
& \bar{\rho}, \bar{J}=\text { characteristic charge and current densities, } \\
& \bar{E}=\text { characteristic electric strengths, } \\
& \bar{B}=\text { characteristic magnetic strengths, }
\end{aligned}
$$

and we set

$$
x=\bar{l} x \prime, \quad t=\bar{t} t \prime, \quad \rho=\bar{\rho} \rho^{\prime} \quad \mathbf{J}=\bar{J} \mathbf{J} /, \quad \mathbf{E}=\bar{E} \mathbf{E} /, \quad \mathbf{B}=\bar{B} \mathbf{B} / .
$$


We assume that the characteristic velocity $\bar{v}$ is small compared with the light velocity c; then Maxwell's equations (1.1)-(1.4) become the following:

$$
\left\{\begin{array}{l}
\frac{\bar{v}}{c^{2}} \overline{\bar{E}} \frac{\partial \mathbf{E}^{\prime}}{\partial t^{\prime}}-\nabla^{\prime} \times \mathbf{B}^{\prime}=-\mu \frac{\bar{J} \bar{l}}{\bar{B}} \mathbf{J} / \\
\bar{v} \frac{\bar{B}}{\bar{E}} \frac{\partial \mathbf{B}^{\prime}}{\partial t^{\prime}}+\nabla^{\prime} \times \mathbf{E}^{\prime}=0 \\
\varepsilon \frac{E}{\bar{l} \rho} \nabla^{\prime} \cdot \mathbf{E}^{\prime}=\rho^{\prime} \\
\nabla^{\prime} \cdot \mathbf{B}^{\prime}=0
\end{array}\right.
$$

The charge conservation equation becomes

$$
\frac{\bar{\rho} \bar{v}}{\bar{J}} \frac{\partial \rho^{\prime}}{\partial t^{\prime}}+\nabla^{\prime} \cdot \mathbf{J}^{\prime}=0
$$

Now given $\bar{l}, \bar{t}$ and $\bar{\rho}$, we choose $\bar{E}$ such that $\varepsilon \frac{\bar{E}}{\bar{l} \bar{\rho}}=1$, and $\bar{J}, \bar{B}$ so that $\frac{\bar{J}}{\bar{\rho}}=c$, $\frac{\bar{E}}{\bar{B}}=c$. Here we set $\eta=\frac{\bar{v}}{c}$, and dropping the primes for simplicity, Maxwell's equations can be written in dimensionless variables as the following:

$$
\begin{gathered}
\eta \frac{\partial \mathbf{E}}{\partial t}-\nabla \times \mathbf{B}=-J, \\
\eta \frac{\partial \mathbf{B}}{\partial t}+\nabla \times \mathbf{E}=0, \\
\nabla \cdot \mathbf{E}=\rho, \\
\nabla \cdot \mathbf{B}=0 .
\end{gathered}
$$

The charge conservation equation turns to be the following:

$$
\eta \frac{\partial \rho}{\partial t}+\nabla \cdot \mathbf{J}=0
$$

with the boundary conditions:

$$
\begin{gathered}
\mathbf{E} \times\left.\mathbf{n}\right|_{\Gamma}=0, \\
\left.\frac{\partial}{\partial t} \mathbf{B} \cdot \mathbf{n}\right|_{\Gamma}=0,
\end{gathered}
$$

and with the initial conditions:

$$
\begin{gathered}
\mathbf{E}(\cdot, 0)=\mathbf{E}_{0}, \\
\mathbf{B}(\cdot, 0)=\mathbf{B}_{0},
\end{gathered}
$$

where $\mathbf{E}_{0}, \mathbf{B}_{0}$ satisfy the following:

$$
\left\{\begin{array}{l}
\nabla \cdot \mathbf{E}_{0}=\rho_{0}, \\
\nabla \cdot \mathbf{B}_{0}=0, \\
\mathbf{E}_{0} \times\left.\mathbf{n}\right|_{\Gamma}=0 .
\end{array}\right.
$$


According to $\nabla \times \mathbf{E}_{L}=0, \mathbf{E}_{L} \times\left.\mathbf{n}\right|_{\Gamma}=0, \nabla \cdot \mathbf{E}_{L}=\rho$, we can get that there exists a function $\phi \in H^{1, *}(\Omega)$ such that $\mathbf{E}_{L}=\nabla \phi$, where $\phi$ satisfies the following:

$$
\left\{\begin{array}{l}
\triangle \phi=\rho, \\
\left.\phi\right|_{\partial \Omega}=-\frac{\frac{1}{\eta} \int_{\Omega} \mathbf{J} \cdot \nabla \chi d x}{\int_{\partial \Omega} \frac{\partial \chi}{\partial \mathbf{n}} d s}, \\
\lim _{|x| \rightarrow \infty} \phi=0 .
\end{array}\right.
$$

From now on, we assume that $\eta$ is a very small parameter. We expand the fields $\mathbf{E}, \mathbf{B}$ in powers of $\eta$.

$$
\begin{aligned}
& \mathbf{E}=\mathbf{E}^{0}+\eta \mathbf{E}^{1}+\eta^{2} \mathbf{E}^{2}+\cdots+o\left(\eta^{k+1}\right), \\
& \mathbf{B}=\mathbf{B}^{0}+\eta \mathbf{B}^{1}+\eta^{2} \mathbf{B}^{2}+\cdots+o\left(\eta^{k+1}\right) .
\end{aligned}
$$

We further assume that $\rho$ is independent of $\eta$, and $\mathbf{J}$ satisfies the following:

$$
\mathbf{J}=\mathbf{J}^{0}+\eta \mathbf{J}^{1}
$$

From the charge conservation equation $\eta \frac{\partial \rho}{\partial t}+\nabla \cdot \mathbf{J}=0$, we know that

$$
\nabla \cdot \mathbf{J}^{0}=0
$$

and

$$
\frac{\partial \rho}{\partial t}+\nabla \cdot \mathbf{J}^{1}=0
$$

We replace (5.2)-(5.5) by $\mathbf{E}, \mathbf{B}, \mathbf{J}^{\prime}$ s expansions (5.13), (5.14), (5.15); then we can obtain formally that $\mathbf{E}^{k}$ and $\mathbf{B}^{k}$ satisfy the following equations:

(i) $o\left(\eta^{0}\right)$ terms:

$$
\begin{aligned}
& \nabla \times \mathbf{B}^{0}=\mathbf{J}^{0}, \\
& \nabla \times \mathbf{E}^{0}=0, \\
& \nabla \cdot \mathbf{E}^{0}=\rho, \\
& \nabla \cdot \mathbf{B}^{0}=0 .
\end{aligned}
$$

(ii) $o\left(\eta^{1}\right)$ terms:

$$
\begin{gathered}
\frac{\partial \mathbf{E}^{0}}{\partial t}-\nabla \times \mathbf{B}^{1}=-\mathbf{J}^{1} \\
\frac{\partial \mathbf{B}^{0}}{\partial t}+\nabla \times \mathbf{E}^{1}=0 \\
\nabla \cdot \mathbf{E}^{1}=0
\end{gathered}
$$




$$
\nabla \cdot \mathbf{B}^{1}=0
$$

(iii) $o\left(\eta^{k}\right)$ terms, $k \geq 2$ :

$$
\begin{gathered}
\frac{\partial \mathbf{E}^{k-1}}{\partial t}-\nabla \times \mathbf{B}^{k}=0, \\
\frac{\partial \mathbf{B}^{k-1}}{\partial t}+\nabla \times \mathbf{E}^{k}=0, \\
\nabla \cdot \mathbf{E}^{k}=0, \\
\nabla \cdot \mathbf{B}^{k}=0 .
\end{gathered}
$$

Similarly, we deduce the following from the boundary condition:

$$
\mathbf{E}^{k} \times\left.\mathbf{n}\right|_{\partial \Omega}=0, \quad k \geq 0 .
$$

We suppose that $\mathbf{B}_{0}$ is independent of $\eta$; then we have

$$
\begin{gathered}
\mathbf{B}^{0} \cdot \mathbf{n}=\mathbf{B}_{0} \cdot \mathbf{n}, \\
\mathbf{B}^{k} \cdot \mathbf{n}=0, k \geq 1 .
\end{gathered}
$$

We assume

$$
\mathbf{E}^{0}(\cdot, 0)=\mathbf{E}_{0 L}=\nabla \phi_{0} .
$$

Theorem 5.5. Assume that the domain $\Omega$ is 3-dimensional and unbounded, $\boldsymbol{J}^{0}$ satisfies (5.16) and $\int_{\partial \Omega} \boldsymbol{J}^{0} \cdot \boldsymbol{n d s}=0, \boldsymbol{J}^{1}$ satisfies (5.17); then we have $\boldsymbol{E}^{0}=\boldsymbol{E}_{L}$.

Proof. From $\nabla \times \mathbf{E}^{0}=0$, we know there exists $\phi^{0}$ such that $\mathbf{E}^{0}=\nabla \phi^{0}$. According to (5.22), arguing as before, we know that $\left(\mathbf{E}^{0}=\nabla \phi^{0}, \mathbf{B}^{1}\right)$ is the solution of the equations (5.19), (5.22), (5.25), (5.30) for $\mathrm{k}=0$ and (5.32) for $\mathrm{k}=1$.

Moreover, combining with (5.17), we know that $\phi^{0}=\phi^{0}(\cdot, t)$ satisfies

$$
\left\{\begin{array}{l}
\frac{\partial}{\partial t}\left(-\triangle \phi^{0}+\rho\right)=0, \\
\left.\frac{\partial \phi^{0}}{\partial t}\right|_{\partial \Omega}=-\frac{\int_{\Omega} \mathbf{J}^{1} \cdot \nabla \chi d x}{\int_{\partial \Omega} \frac{\partial \chi}{\partial \mathbf{n}} d s}, \\
\lim _{|x| \rightarrow \infty} \phi^{0}=0 .
\end{array}\right.
$$

If we assume $\phi^{0}(\cdot, 0)$ satisfying $\triangle \phi^{0}(\cdot, 0)=\rho(\cdot, 0)$, we can get $\nabla \cdot \mathbf{E}^{0}=\rho$, i.e., $\phi^{0}$ satisfies

$$
\triangle \phi^{0}=\rho .
$$

On the other hand, from $\nabla \cdot \mathbf{J}^{0}=0, \int_{\partial \Omega} \mathbf{J}^{0} \cdot \mathbf{n} d s=0$ we have

$$
\int_{\Omega} \mathbf{J}^{0} \cdot \nabla \chi d x=-\int_{\Omega} \nabla \cdot \mathbf{J}^{0} \chi d x+\int_{\partial \Omega} \mathbf{J}^{0} \cdot \mathbf{n} \chi d s=0 .
$$


Since $\mathbf{J}=\mathbf{J}^{0}+\eta \mathbf{J}^{1}$, we have the following:

$$
\int_{\Omega} \mathbf{J}^{1} \cdot \nabla \chi d x=\frac{1}{\eta} \int_{\Omega} \mathbf{J} \cdot \nabla \chi d x
$$

Combining with (5.34), (5.35), (5.36), we know $\phi^{0}$ coincides with the solution of (5.12), i.e., $\mathbf{E}^{0}=\mathbf{E}_{L}$.

Now let us consider the Darwin model in scaled variables

$$
\left\{\begin{array}{l}
\eta \frac{\partial \mathbf{E}_{L}^{D}}{\partial t}-\nabla \times \mathbf{B}^{D}=-J \\
\eta \frac{\partial \mathbf{B}^{D}}{\partial t}+\nabla \times \mathbf{E}_{T}^{D}=0 \\
\nabla \cdot \mathbf{E}_{L}^{D}=\rho \\
\nabla \cdot \mathbf{E}_{T}^{D}=0 \\
\nabla \times \mathbf{E}_{L}^{D}=0 \\
\nabla \cdot \mathbf{B}^{D}=0
\end{array}\right.
$$

provided with the boundary condition (4.17-4.20) and the initial condition $\mathbf{E}_{L}^{D}(\cdot, 0)=\mathbf{E}_{0 L}$.

TheOrEm 5.6. Assume the hypotheses of Theorem 5.5; then we have $\boldsymbol{E}_{L}^{D}=\boldsymbol{E}^{0}, \boldsymbol{E}_{T}^{D}=$ $\eta \boldsymbol{E}^{1}+\eta^{2} \boldsymbol{E}^{2}, \boldsymbol{B}^{D}=\boldsymbol{B}^{0}+\eta \boldsymbol{B}^{1}$.

Proof. We have proved that $\mathbf{E}_{L}^{D}=\mathbf{E}_{L}=\mathbf{E}^{0}$.

Now we consider $\widetilde{\mathbf{E}_{T}^{D}}=\eta \mathbf{E}^{1}+\eta^{2} \mathbf{E}^{2}, \widetilde{\mathbf{B}^{D}}=\mathbf{B}^{0}+\eta \mathbf{B}^{1}$. Using (5.18)-(5.29), we can check that $\left(\mathbf{E}_{L}, \widetilde{\mathbf{E}_{T}^{D}}, \widetilde{\mathbf{B}^{D}}\right)$ is indeed a solution of the equations (5.37). Moreover, it satisfies the boundary condition (4.17)-(4.20) and the initial condition $\mathbf{E}_{L}^{D}(\cdot, 0)=\mathbf{E}_{0 L}$ by (5.30)-(5.32) and (5.33) respectively. According to the well-posedness of the Darwin model in Fang and Ying [3], we know that $\mathbf{E}_{T}^{D}=\widetilde{\mathbf{E}_{T}^{D}}, \mathbf{B}^{D}=\widetilde{\mathbf{B}^{D}}$. Hence $\mathbf{E}_{L}^{D}=\mathbf{E}^{0}$, $\mathbf{E}_{T}^{D}=\eta \mathbf{E}^{1}+\eta^{2} \mathbf{E}^{2}, \mathbf{B}^{D}=\mathbf{B}^{0}+\eta \mathbf{B}^{1}$ holds.

TheOREM 5.7. Assume that the hypotheses of Theorem 5.6 hold; then we have the following error estimates: $\left\|\boldsymbol{E}-\boldsymbol{E}^{D}\right\|_{0, \Omega} \leq C \eta^{3}$ and $\left\|\boldsymbol{B}-\boldsymbol{B}^{D}\right\|_{0, \Omega} \leq C \eta^{2}$.

The proof of the theorem is obvious.

Acknowledgement.We are grateful to the referees for their detailed comments to improve the paper, in particular their calling our attention to the paper by Raviart and Sonnendrücker.

\section{REFERENCES}

[1] L.A. Ying, Numerical Methods for Exterior Problems, World Scientific, Singapore, 2006.

[2] O.A. Ladyzhenskaya, The Mathematical Theory of Viscous Incompressible Flow., Gordon and Breach, second edition, New York, 1969.

[3] N.S. Fang and L.A. Ying, Three dimensional exterior problem of Darwin model and its numerical computation, preprint.

[4] R.A. Adams, Sobolev Spaces, Academic Press, New York, 1975.

[5] P. Degond and P.A. Raviart, An analysis of the Darwin model of approximation to Maxwell's equations, Forum Math., 4, 13-44, 1992.

[6] P. Ciarlet and J. Zou, Finite element convergence for the Darwin model to Maxwell's equations, RAIRO Math. Modelling Numer. Anal., 31, 213-249, 1997.

[7] C.X. Liao and L.A. Ying, The equivalence between the TE, TM model and the Darwin models in 2-D bounded multiply connected domains and 2-D unbounded domains, preprint.

[8] P.A. Raviart and E. Sonnendrücker, $A$ hierarchy of approximate models for the Maxwell equations, Numer. Math., 73, 329-372, 1996. 